\title{
THE IPS WORK IN BEIJING ASTRONOMICAL OBSERVATORY
}

\author{
QIU YUHAI* \\ Beijing Astronomical Observatory \\ Chinese Academy of Sciences, Beijing 100080, China
}

\begin{abstract}
This article describes the IPS work in the Beijing astronomical observatory including the hardware and software of the instrument and some theoretical research.
\end{abstract}

Key words: IPS - Radio Interferometer - Data handling

\section{Introduction}

There is a small group in BAO to do the IPS research.By means of Miyun compound interferometer the IPS observation will be started in near future. Preliminary observation of a few radio sources has been done to check the performance of the instrument.Some theory work is in progress too.

\section{Hardware}

The Miyun aperture synthesis radio telescope (Wang. S.G. et al. 1985) working at 232 and $327 \mathrm{MHz}$ is an array of $192(16 * 12)$ simultaneous interferometer formed by 28 fixed $9 \mathrm{~m}$ steerable antennae on E-W baseline. This array is divided into $\mathrm{A}$ and $\mathrm{B}$ sub-array which consist of 16 and 12 same dishes respectively (see fig.1). By means of the new backend the synthesis telescope has been made a compound interferometer that will be used for IPS observation. The main features of this system are :

$\begin{array}{ll}\text { observing frequency } & 232 \& 327 \mathrm{MHz} \\ \text { aerials } & 28 \times 9 \mathrm{~m} \text { parabolic dishes } \\ \text { maximun length of E-W base-line } & 1242 \mathrm{~m} \\ \text { total physical area } & 1770 \mathrm{~m}^{2} \\ \text { east-west beam width } & 3.6^{\prime}(\mathrm{fan} \text { bean }) \\ \text { band width } & 1.5 \mathrm{MHz} \\ \text { sensitivity (232MHz) } & 2.7 J y \cdot(1 \mathrm{sec} . \text { integration period) } \\ \text { phase compensation minimum step } & 5.6^{\circ}\end{array}$

At the $232 \mathrm{MHz}$ system the IF output signals $(30 \mathrm{MHz})$ of each antenna in synthesis system are mixed with the second LO signal $(40.7 \mathrm{MHz})$ and then

* The project is supported by the China National Nature Science Fundation under the program 49391400 


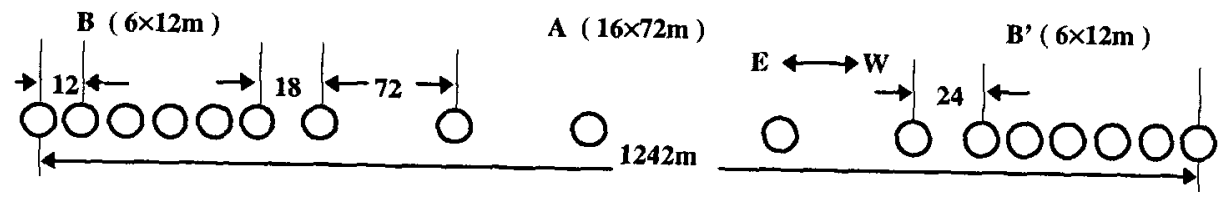

Fig. 1. The configration of Miyun array

the output signals $(10.7 \mathrm{MHz})$ are shifted by proper phase,respectively,via 28 channels phase-shifter to compensate for the phase difference between each antenna and the phase change due to the earth rotation. The signals from A sub-array (16 dishes) and the B sub-array (12 dishes) are added together respectively and the two output signals are then correlated. The detected output is smoothed with $40 \mathrm{~ms}$ RC low-pass filter and sent to an on-line computer via a suitable interface, then sampled at $50 \mathrm{~Hz}$ via $\mathrm{A} / \mathrm{D}$ converter to produce the observing output.

\section{Software}

A set of program has been developed (Qiu Y.H. 1992) under the microcomputer and sun workstation to do all the tasks as the following :

\subsection{ON-LINE SOFTWARE}

a) Real-time observation program for IPS: It performs four main tasks in on-line operation: inputting to the computer essential data of the observation and starting the observation at the right start time; calculating the phase change due to the earth rotation for each antenna and control of 28 channeis of the phase shifter to stop the phase fringe; sampling the output data every $20 \mathrm{~ms}$; recording and display of the observed data in real-time.

b) Calibration observation program : By means of observing the calibration of radio source such as Cyg. A, the phases and amplitudes of every antenna could be deduced. This set of data was used for system correction.

c) Scan observing program : This program is used for scan observation of sun or other radio sources in proper scan rate. The fastest rate is $20 \mathrm{~ms}$ per point.

\subsection{OFF-LINE SOFTWARE}

a) Radio source database for IPS observation: From the radio source catalogue of Cambridge for IPS observation (Purvis A., 1987) 44 radio 

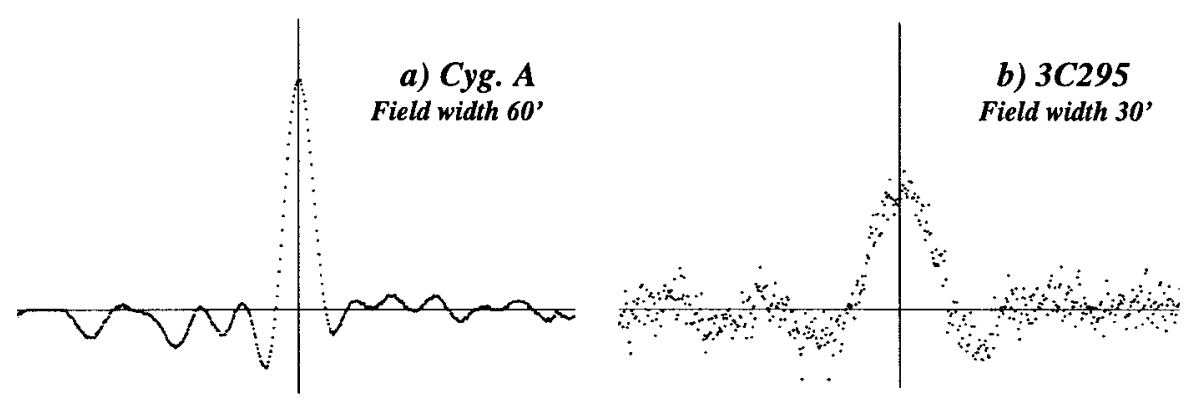

Fig. 2. One dimension scan of Cyg.A and 3C295

sources have been chosen for our observation according to the following standard : flux $>5 J y$ and angular diameter $<0.2$ ".

b) Redisplay,edit and sample of the data : All the data could be re-displayed and edited to check the observation quality and to reject the bad data (spikes or sudden changes). The data also could be resampled for further processing. [Usually all the data was convolved with a rectangular window and resampled at $40 \mathrm{~Hz}$.]

c) The scintillation index calculation: The scintillation index could be calculated by the formula as below:

$M=\langle\Delta I\rangle /\langle I\rangle=\left\{\sigma_{o n}-\sigma_{o f f}\left(1+T_{s} / T_{o f f}\right)\right\} / T_{s}$

where $T_{s}=T_{o n}-T_{o f f}, T_{o n}$ and $\sigma_{o n}$ are the antenna temperature and the standard deviation "on" source respectively ; $T_{\text {off }}$ and $\sigma_{o f f}$ are the same, but "off" source.

d) The IPS spectra calculation: The mean of each data block (512 points) was subtracted and multiplied by a triangular weighting function, which is unity at the center falling to zero at both ends, and then transformed using FFT. The auto correlation spectra was formed by multiplying the transformed series by its conjugate. The resolution of resulting spectra is about $0.16 \mathrm{~Hz}$.

\section{Preliminary observation results of some radio sources}

Some radio sources have been observed for the performance test of this instrument such as Cyg.A, 3C123, 3C219, 3C295 and 3C433 in May 1994. These results are obtained by scanning radio sources, $20 \mathrm{~ms}$ per point and shown in figure 2. The left of fig.2 is the convolved result of the response of the compound interferometer on Cyg.A and shows that there is slight asymmetry and deeper negative sidelobe which may be caused by the imperfect calibration of phase and amplitude, non-uniform aperture illumination and the rough step of phase compensation. Due to the extension of Cyg.A 
(about 2.1') the primary power pattern of the instrument is narrower than the beam of Fig. 2 but could be deduced easily. From other observation it is also easy to determine the sensitivity of this interferometer by known flux of the radio source. All of this shows that the instrument performances such as the primary power pattern and sensitivity are about the same as predicted by theory.

\section{Theory work}

A series of papers (Ma G.Y. 1993a,1993b,1994) about scintillation theory have been published . On the basis of phase screen theory by using numerical integration, these papers discuss the effect of the inner-scale, outer-scale and the anisotropic behaviors of electron density irregularities on the intensity scintillation spectra under the assumption of those spatial spectrum following a power law . Some numerical analogous work of scintillation spectra have been done to compare with the scintillation measurements.

\section{The future plan}

Because of the man-made interference in $232 \mathrm{MHz}$ all the frontends are updated now to suppress the interference. If the man-made interference could be suppressed successfully by the new preamplifier, IPS observation could be begun in near future. In the proposal of the Chinese space physics development program for the period of the next 10 years the 3 -station IPS observation instrument such as the Japan system has been suggested to establish at Beijing but it all depends on the financial support of our government.

\section{References}

Ma G.Y.1993a A Preliminary Computation Result of Plasma Irregularities in Solar WindC.Y.Jiang et. al., ISPR' 93 Beijing,CHINA 259.

Ma G.Y., Wang J. L.:1994, 'Research of solar Wind by Observations of Radio Wave Propagation' ,Progress in Astronomy (China) 1.12., 30.

Ma G.Y. .:1993b, 'A Study of Interplanetary Scintillation and Its Application', Thesis for PH.D. Degree, in Astrophysics

Purvis, A. et al .:1987, 'The Cambridge IPS survey at $81.5 \mathrm{MHz}$ 'Mon. Not. R .Astr. Soc.229, 589.

Qiu Yuhai ,:1993, 'Software for IPS Work and IPS data Handling Sub-Package under IRAF' The publication of Beijing astronomical observatory .20,82.

Wang,S.G. et al.:1985, 'The Miyun Metre-wave Aperture Synthesis Radio Telescope', Acta Astrophysics. Sin.10.5., 245. 